\title{
Überlebensverlängerung auch bei rezidivierten oder metastasierten Tumoren
}

Nahezu alle Plattenepithelkarzinome im Kopf-Hals-Bereich exprimieren den Rezeptor für den epidermalen Wachstumsfaktor (EGFR). Bei rezidivierten oder metastasierten Tumoren dieser Art hatte es in den letzten 25 Jahren keine Therapie geschafft, die Überlebenszeiten unter dem bisherigen Standard, einer platinbasierten Chemotherapie, zu verlängern. Dem EGFR-Antikörper Cetuximab (Erbitux ${ }^{\circledR}$ ) ist dies nun in einer großen internationalen Studie in Kombination mit der StandardChemotherapie erstmals gelungen.

Cetuximab ist derzeit in Kombination mit Strahlentherapie zur Behandlung von $\mathrm{Pa}$ tienten mit lokal fortgeschrittenem Plattenepithelkarzinom zugelassen. Grundlage für die Zulassung war eine große Phase-III-Studie, in der Cetuximab die Überlebensdauer im Vergleich zu alleiniger Strahlentherapie signifikant von 29,3 auf 49 Monate verlängerte. Rezidivierte oder metastasierte Stadien dieser Tumoren sind besonders schwer zu therapieren. In einer weiteren großen PhaseIII-Studie, der EXTREME-Studie (ErbituX in first line Treatment of REcurrent or MEtastatic head \& neck cancer), wurde untersucht, ob Cetuximab auch hier zu einer Überlebensverlängerung im Vergleich zur Standardtherapie führen kann.

In die Studie, so Professor Hansjochen Wilke, Essen, auf der Jahrestagung der Deutschen, Österreichischen und Schweizerischen Gesellschaften für Hämatologie und Onkologie, waren 442 Patienten eingeschlossen. Diese erhielten eine Kombination aus Cisplatin $\left(100 \mathrm{mg} / \mathrm{m}^{2}\right.$ an Tag 1) bzw. Carboplatin (AUC 5) und 5-Fluorouracil $\left(1000 \mathrm{mg} / \mathrm{m}^{2}\right.$ an den Tagen $1-4$, Zyklusdauer 3 Wochen). Randomisiert bekam die Hälfte der Patienten zusätzlich entweder Cetuximab $\left(400 \mathrm{mg} / \mathrm{m}^{2}\right.$ als Initialdosis und danach wöchentlich $250 \mathrm{mg} / \mathrm{m}^{2}$ ) oder Placebo über sechs Zyklen bzw. bis zum Auftreten von Tumorprogression oder inakzeptablen Toxizitäten. Zusätzlich konnten die Patienten in der Verumgruppe nach Abschluss der Kombinationstherapie aus Cetuximab und platinba- sierter Chemotherapie eine Erhaltungstherapie nur mit Cetuximab bekommen. Die Ergebnisse der EXTREME-Studie waren zum ersten Mal bei der diesjährigen ASCO-Jahrestagung in Chicago präsentiert worden. Die zusätzliche Gabe von Cetuximab erzielte einen Vorteil bei allen Effektivitätsparametern, so Wilke [1]:

- Die objektive Ansprechrate wurde durch Cetuximab nahezu verdoppelt - von $19,5 \%$ auf $35,6 \%(\mathrm{p}<0,0001)$.

- Cetuximab verlängerte das mediane progressionsfreie Überleben von 3,3 auf 5,6 Monate. Die Hazard Ratio für das progressionsfreie Überleben lag bei 0,538 , d.h. das Risiko für eine Progression konnte durch die Kombination aus Cetuximab und platinbasierter Chemotherapie um insgesamt $46 \%$ reduziert werden $(p<$ 0,0001).

- Auch der härteste onkologische Effektivitätsparameter, die mediane Gesamtüberlebenszeit, wurde durch Cetuximab signifikant von 7,4 auf 10,1 Monate verlängert. Die Hazard Ratio für das Überleben betrug 0,797 ( $p<0,0362$ ), das Mortalitätsrisiko ist also um rund $20 \%$ gesenkt.

Nachdem es für Patienten mit rezidivie- renden und metastasierten Kopf-Hals-Tumoren über viele Jahre hinweg keine wirksamen Behandlungsoptionen gegeben habe, sei es mit Cetuximab nun erstmals seit 25 Jahren gelungen, die Überlebenszeit signifikant $\mathrm{zu}$ verlängern, zitierte Wilke den EXTREME-Studienleiter Professor Jan Vermorken, Antwerpen (Abb. 1).

Die Lebensqualität der Patienten, die zu Beginn, nach 3 und nach 6 Monaten mittels Fragebogen ermittelt worden war, hatte sich durch die Antikörpertherapie nicht signifikant verschlechtert. Abgesehen von den für Cetuximab zu erwartenden Hautausschlägen und Reaktionen an der Infusionsstelle wurden unter der zusätzlichen Antikörpertherapie nicht mehr Toxizitäten registriert als in der Gruppe, die nur Chemotherapie erhalten hatte.

Die EXTREME-Studie hat damit einen neuen Standard für die Behandlung rezidivierter oder metastasierter Plattenepithelkarzinome im Kopf-Hals-Bereich definiert, so Professor Ulrich Keilholz, Berlin, in seinem anschließenden Vortrag.

Auch die Kombination von Cetuximab mit Taxanen scheint ein vielverspre-

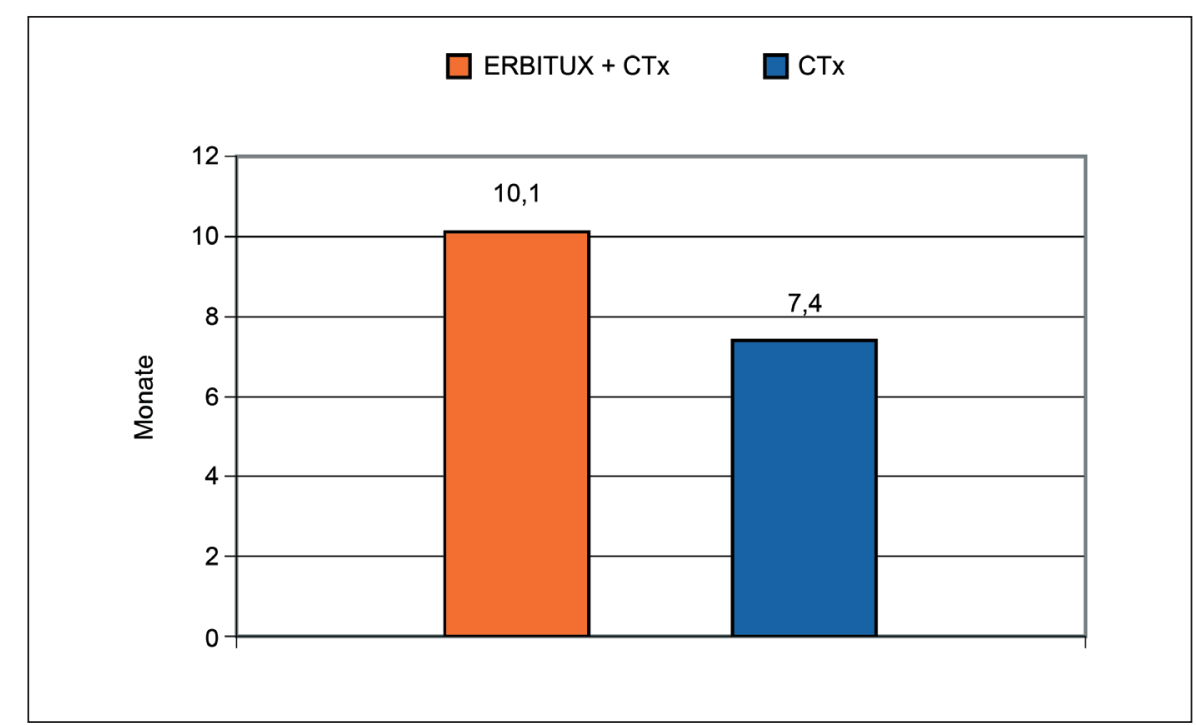

Abb. 1. Signifikante Verlängerung des medianen Überlebens durch Cetuximab in Kombination mit einer platinbasierten Chemotherapie im Vergleich zu Chemotherapie allein. 


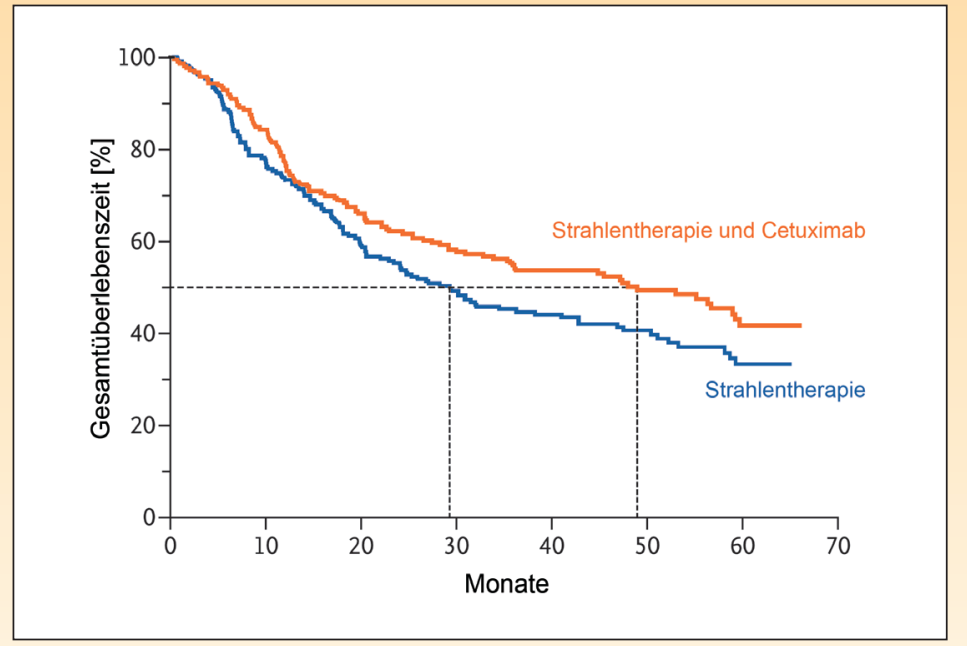

Abb. 2. Überlebenskurven der $\mathrm{Pa}$ tienten in der Zulassungsstudie [nach Bonner et al. $\mathrm{N}$ Engl J Med 2006; 354: 567-78]

chender Ansatz in der Behandlung von rezidivierten und/oder metastasierten KopfHals-Tumoren zu sein, wie neue Daten vom ASCO zeigen. Der Benefit, der durch die Cetuximab-Therapie erzielt wird, könnte so noch weiter ausgebaut werden. Deshalb, so Keilholz, gaben diese ersten Ergebnisse den Anlass, an der Berliner Charité eine Phase-II-Studie zu initiieren, in der zur Zeit die Kombination aus Docetaxel und Cetuximab getestet wird. Bereits in Vorbereitung ist ein weiteres PhaseII-Nachfolgeprojekt für die EXTREME-Studie, in dem Patienten mit rezidivierten oder metastasierten Tumoren randomisiert die Kombination aus Cetuximab/Cisplatin/5-FU mit oder ohne Docetaxel erhalten sollen.

\section{Lokoregionär fortgeschrittene Kopf-Hals-Tumore:}

Die Strahlentherapie ist eine Standardbehandlung des lokal fortgeschrittenen Plattenepithelkarzinoms im Kopf-Hals-Bereich. In Kombination mit Chemotherapie ist sie noch wirksamer, aber so nebenwirkungsreich, dass sie von den Patienten oft nicht toleriert wird, so Professor Jürgen Dunst, Lübeck, in seinem Vortrag. Vor allem eine Grad-3/4-Mukositis setzt diesem konventionellen Therapieschema enge Grenzen.

Die Kombination aus Strahlentherapie und Cetuximab hatte sich in präklinischen Untersuchungen als synergistisch erwiesen. Dies war die Rationale für eine der bisher größten Phase-III-Studien in dieser Indikation. 424 Patienten mit lokoregionär fortgeschrittenen, inoperablen Plattenepithelkarzinomen des Stadiums III oder IV erhielten in kurativer Intention eine Hochdosis-Strahlentherapie (70-76,8 Gy fraktioniert oder hyperfraktioniert zweimal täglich oder in Form eines concomitant boosts, je nachdem, wie es im jeweiligen Zentrum gebräuchlich war). Die Hälfte der Patienten erhielt zusätzlich Cetuximab (initial $400 \mathrm{mg} / \mathrm{m}^{2}$, dann wöchentlich $250 \mathrm{mg} / \mathrm{m}^{2}$ für die Dauer der Radiotherapie [2]).

Die Gesamtuiberlebenszeit wurde durch die zusätzliche Gabe von Cetuximab von median 29,3 Monaten auf 49 Monate beinahe verdoppelt (HR für Tod $0,74, \mathrm{p}=$ 0,03 , d.h. das Mortalitätsrisiko war signifikant um 26\% reduziert). Zusätzlich war die lokoregionäre Kontrolle mit median 24,4 Monaten im Cetuximab-Kombinationsarm im Vergleich zu lediglich 14,9 Monaten in der Kontrollgruppe (Hazard Ratio für lokoregionäre Progression oder Tod $0,68, p=0,005)$ signifikant verlängert. Die Kurven sowohl für Krankheitskontrolle als auch für das Überleben verliefen noch nach 4 Jahren parallel, d.h. der Effekt der mehrwöchigen CetuximabBehandlung hält über Jahre hinweg an (Abb. 2). Aufgrund dieser Ergebnisse wurde Cetuximab im April 2006 von der EMEA in Kombination mit einer Strahlentherapie für die Behandlung von lokal fortgeschrittenen Plattenepithelkarzinomen des Kopfes und Halses zugelassen.

Die zusätzliche Gabe von Cetuximab führte bis auf die bekannten Nebenwirkungen wie akneformen Hautauschlag in dieser Studie zu keiner Erhöhung der strahlentherapiebedingten Nebenwirkungen. Insbesondere die Häufigkeit einer Grad-3/4-Mukositis war in beiden Behandlungsgruppen annähernd gleich.
Die Anti-EGFR-Therapie zusätzlich zur Hochdosis-Strahlentherapie kann also nicht nur die lokoregionäre Krankheitskontrolle, sondern auch das Gesamtüberleben bei Plattenepithelkarzinomen im Kopf-Hals-Bereich deutlich und signifikant erhöhen, ohne die Nebenwirkungen der Strahlentherapie oder ihre Letalität zu verstärken, so Dunst.

Auch die Lebensqualität war, wie eine neue Analyse der Studie zeigt [3], durch die Zugabe von Cetuximab im Vergleich zur alleinigen Strahlentherapie nicht verschlechtert: Sie wurde mit dem EORTCLebensqualitäts- und dem Kopf-HalsTumor-spezifischen Fragebogen erhoben und nahm in beiden Gruppen zunächst $a b$, um sich nach Ende der Therapie wieder zu verbessern; nach $1 \mathrm{Jahr}$ waren die Ausgangswerte wieder erreicht. Interessanterweise hing das Überleben stark von der Lebensqualität zu Studienbeginn ab.

\section{Josef Gulden, Grafrath}

\section{Quelle}

Satellitensymposium «Erbitux ${ }^{\circledR}$ - neue Hoffnung in der Therapie von Kopf-Hals-Tumoren» bei der Jahrestagung der Deutschen, Österreichischen und Schweizerischen Gesellschaft für Hämatologie und Onkologie (DGHO) in Basel, 5. Oktober 2007.

\section{Literatur}

1 Vermorken JB et al. Cetuximab plus platinum-based therapy first-line in recurrent and/or metastastic (R/M) squamous cel carcinoma of the head and neck (SCCHN): Efficacy and safety results of a randomized phase III trial (EXTREME). Eur J Cancer 2007:5:324, abstr \#5501

2 Bonner JA et al. Radiotherapy plus cetuximab for squamouscell carcinoma of the head and neck. N Engl J Med 2006; 354: $567-78$

3 Curran D et al. Quality of life in head and neck cancer patients after treatment with high-dose radiotherapy alone or in combination with cetuximab. J Clin Oncol 2007;25:2191-7.

\section{Impressum}

Cetuximab bei Kopf-Hals-Tumoren

Überlebensverlängerung auch bei rezidivierten oder metastasierten Tumoren

PharmaForum in ONKOLoGIE 30 | 12 | 07

C 2007 by S. Karger Verlag für Medizin und Naturwissenschaften $\mathrm{GmbH}$

Lörracher Straße 16a

79115 Freiburg, Deutschland

\section{Mit freundlicher Unterstützung durch} die Merck Pharma GmbH.

Der Verlag und die Herausgeber der Zeitschrift übernehmen keine Verantwortung für diese Rubrik.

\section{KARGER ๑) 2007 S. Karger GmbH, Freiburg}

